\title{
Integrando La Música En La Educación Infantil A Través Del Equipo Docente Interdisciplinar
}

\author{
Rosa María Serrano Pastor \\ University of Zaragoza, Spain
}

doi: 10.19044/esj.2017.v13n7p347 URL:http://dx.doi.org/10.19044/esj.2017.v13n7p347

\begin{abstract}
School education is a complex process in which students and teachers interact in a context. In order to obtain an adequate and complete advance in the field of educational research, it is necessary to study in depth the different parts of this process. This article focuses on the study of the interdisciplinary teamwork of teachers in a project on the awareness of the linguistic discourse, verbal and musical, in early childhood education. In this project the generalist teachers carried out the music-verbal activities with their students. The objective of this study was to analyse the influence of the work in teaching team, composed by generalist teachers and music specialist, in this project; examining the parameters that influence its functioning and observing the benefits of its application. The analysis has been carried out from the qualitative methodology, finding that previous knowledge and experience of each teacher, positive interdependence between them, shared responsibility and the role of the music specialist and of the School influence the proper functioning of this group. The teamwork carried out in this project has reported benefits, among which are the production of linguistic knowledge, training and professional development of the teachers involved. It is valued that the project is not only beneficial for students but also for teachers.
\end{abstract}

Keywords: Teaching team, Music education, Childhood education, Interdisciplinarity, Music.

\section{Resumen}

La educación escolar es un proceso complejo en el que interaccionan alumnado y profesorado en un contexto determinado. Para obtener un adecuado y completo avance en el ámbito de la investigación educativa, es necesario estudiar en profundidad las diferentes partes de dicho proceso. Este artículo se centra en el estudio del trabajo en equipo interdisciplinar del profesorado en un proyecto sobre la toma de conciencia del discurso 
lingüístico, verbal y musical, en la etapa infantil, en el que los propios maestros tutores llevaron a cabo las actividades músico-verbales con su alumnado. El objetivo de este estudio ha sido analizar la influencia del trabajo en equipo docente, compuesto por tutores y especialista de música, en dicho proyecto; examinando los parámetros que influyen en su funcionamiento y observando los beneficios de su aplicación. El análisis se ha realizado desde la metodología cualitativa, encontrando que el conocimiento y experiencia previas de cada docente, la interdependencia positiva entre ellos, la responsabilidad compartida y la labor del especialista de música y del Centro influyen en el buen funcionamiento del mismo. El trabajo en equipo realizado en este proyecto ha reportado beneficios, entre los que se destacan la producción de conocimiento lingüístico, la formación y el desarrollo profesional de los docentes involucrados. Se valora que el proyecto no solo es beneficioso para el alumnado sino también para el profesorado implicado en el equipo.

Palabras clave: Equipo docente, Educación musical, Educación Infantil, Interdisciplinariedad, Música.

\section{Introducción}

La educación escolar es un proceso complejo a través del cual se busca que el individuo adquiera ciertos conocimientos que son esenciales para su desarrollo personal y social. En dicho procedimiento ocupa un rol de vital importancia tanto el proceso de enseñanza como de aprendizaje, así como los participantes en el mismo, alumnado, profesorado y el contexto en el que se desarrolla. Cada uno de estos integrantes no actúa solo sino en relación con otros, influyendo de manera decisiva los vínculos que entre ellos se desarrollan. En España, la enseñanza en la Educación Infantil la realizan maestros formados para impartir en esta etapa, además de la intervención puntual de especialistas en áreas específicas, como es el caso de la música. Dicha intervención no es obligatoria sino que depende de la disponibilidad del Centro y de dicho especialista, siendo en muchos casos el propio tutor el que debe integrar la música en el proceso educativo. Este escenario hace que la colaboración e intercambio de información, conocimientos y metodologías entre profesorado tutor y especialista de música debería ser una realidad que mejore el proceso de enseñanza-aprendizaje. El trabajo en equipo docente interdisciplinar puede convertirse en una herramienta con un gran potencial en los Centros educativos.

Este artículo se centra en estudiar el funcionamiento del profesorado al trabajar en equipo colaborativo en un contexto innovador. En concreto, se expone el análisis del trabajo en equipo interdisciplinar entre docentes de educación infantil y especialista de música en un proyecto llevado a cabo en 
un Colegio Público de Infantil y Primaria, donde eran los tutores los que llevaban a la práctica con el alumnado de infantil todo el desarrollo del mismo. Este estudio forma parte de un trabajo mayor, circunscrito en la toma de conciencia del discurso lingüístico, verbal y musical, en la etapa infantil, en el que se han analizado los diferentes elementos implicados en el proceso educativo: profesorado, alumnado y proceso de enseñanza-aprendizaje del discurso lingüístico. Los resultados que aquí se presentan se centran en el profesorado, en concreto en el análisis del trabajo en equipo colaborativo docente, examinando los factores que repercuten en el funcionamiento de dicho equipo así como los beneficios obtenidos.

\section{Fundamentos teóricos}

\section{El equipo colaborativo}

El trabajo en equipo permite que una persona compense a otra y que todos agudicen su ingenio para resolver cuestiones que conciernen a todos. En el trabajo de equipo se toman decisiones y se actúa sobre ellas. Esta es la finalidad del equipo. En esa actuación, todos adquieren una responsabilidad ante el colectivo. Además, el propio equipo evalúa el proceso, revisa las decisiones y las corrige o las cambia (Armengol, 2002, p. 15).

Las palabras de esta autora resumen el sentido de un equipo colaborativo en un centro educativo. Resalta las bases sobre las que se debe asentar dicho grupo si realmente se quiere realizar un proceso y obtener un resultado satisfactorio y óptimo. Entre las ventajas indicadas cabría destacar la participación en las decisiones, la satisfacción personal, el compromiso con la institución, el incremento de las informaciones y los puntos de vista respecto de las situaciones del alumnado y el centro, la coherencia en la formación del alumnado y la mejora, en definitiva, de la calidad educativa.

El enfoque de colaboración entre docentes se define como la indagación práctica realizada por el profesorado, de forma colaborativa, con la finalidad de mejorar su práctica educativa a través de ciclos de acción y reflexión (Latorre, 2003). Esta colaboración se realiza en equipo y debe contar con una interdependencia positiva entre sus integrantes. De esta manera se pueden establecer vínculos de unión en la búsqueda de objetivos compartidos, en la coordinación de esfuerzos por ver cumplidos esos objetivos y en la periódica retroalimentación basada en la experiencia práctica para la mejora en el proceso. De la calidad de la labor de equipo desarrollada dependerá la calidad de los procesos y los resultados educativos (Bonals, 1996).

Entre las bonanzas de este trabajo conjunto se puede destacar el acceso a nuevas ideas, el establecimiento de relaciones personales más positivas, la estimulación de la creatividad, la adaptación del currículo al contexto y a las necesidades del alumnado y el aumento de las oportunidades que los 
profesores tienen para aprender y avanzar en su propio desarrollo profesional (López, 2007). Otros autores como Bonals (1996) destacan entre sus beneficios la unificación de criterios en la tarea docente, ofreciendo un sentido más global del proceso de enseñanza-aprendizaje a la comunidad educativa y una mayor calidad de enseñanza. Subraya la facilitación de intercambio de experiencias docentes que se hace posible gracias a la reflexión colectiva de la experiencia que tiene cada uno de su práctica docente, obteniendo una mejora en el análisis de problemas y en la elaboración de respuestas a dichos problemas. Asimismo se consigue una mayor identificación del docente con el grupo de trabajo con el objetivo común de mejorar las prácticas educativas. Los docentes desarrollan una mayor coordinación y colaboración, promoviendo la renovación y mejora de sus actitudes y actuaciones, y en general la satisfacción en su tarea.

Sin embargo, pese a todos estos beneficios contrastados, la concienciación de que la educación es una tarea colectiva y que en el centro educativo se pueden llevar a cabo propuestas muy interesantes que beneficien tanto al profesorado como al alumnado es una idea que ha sufrido una evolución muy lenta. Todavía hoy en muchos colegios existe gran reticencia a su puesta en marcha. Para que se dé realmente un acto de colaboración docente se necesita de la decisión y esfuerzo de todos los implicados (Armengol, 2002).

Entre las dificultades más habituales encontradas a la hora de trabajar en equipo se podrían destacar las inhibiciones iniciales, los silencios molestos, la participación solamente de una parte de los miembros y ciertas actitudes poco predispuestas al trabajo grupal (Bonals, 1996). Los problemas en la secuenciación de los turnos de intervención es otra de las complicaciones más habituales. Estos pueden llevar a algunos miembros a sentirse molestos o a evitar participar. Suelen afectar las paralizaciones del trabajo por polarizaciones de las ideas de los miembros, por centralizaciones en detalles cuando no es el momento o salidas del tema, o los desequilibrios exagerados y constantes en las sesiones en la participación y toma de decisiones. Estos con frecuencia desembocan en malestar de cierta parte del grupo por sentirse manipulados o en inhibiciones por miedo a la crítica. El excesivo número de integrantes, la repartición inoperante del trabajo, el uso inapropiado de técnicas de trabajo, la articulación poco adecuada de las diversas partes del trabajo o una temporalización inadecuada a menudo influyen negativamente en el progreso del trabajo de equipo. A estos factores con frecuencia se unen dificultades añadidas por el propio centro, que impide contar con tiempo para las reuniones o autonomía del equipo para decidir qué contenidos trabajar y cómo hacerlo (López, 2007). Ampliando el análisis de las dificultades del trabajo en equipo, Antúnez (1999) señala como factores arraigados las prácticas educativas individualistas tradicionales en la gran mayoría de los 
centros que llevan al aislamiento y a la falta de coordinación. La división del propio centro en departamentos, ciclos u otro tipo de unidades no favorecen la relación. Tampoco lo hacen unos horarios escolares rígidos que impiden los encuentros, intercambios y el trabajo de colaboración, así como una baja disposición del profesorado a dichas coordinaciones. La convicción del profesorado ante la idea de que el trabajo individualista implica no tener que dar explicaciones y no perjudica el futuro profesional del enseñante y una falta de formación del profesorado para ser miembros eficaces de una organización, a menudo se convierten en razones que frenan este proceso grupal.

\section{La música en el currículo de infantil}

A este marco general descrito, se le une la falta de formación específica sobre la integración de la música en el currículo general que dificulta esa colaboración en equipo entre maestros tutores y especialista de música. Los estudios realizados sobre la incorporación musical al currículo infantil por el profesor tutor dan muestras de que esta ha sido mínima y que la mayoría de los profesores no se encuentran a gusto enseñando música (Bresler, 1993). La periodicidad con la que la mitad del profesorado generalista utiliza la música es de una vez al mes (McCarthy, 1993) y el tiempo de su uso suele ser muy limitado (Saunders y Baker, 1991). Las actividades que habitualmente se desarrollan son especialmente la de cantar, seguidas de otras como la escucha de música, las actividades de movimiento, como danzas tradicionales, y la relación con otras asignaturas (Bryson, 1982; Saunders y Baker, 1991). Las actividades encaminadas al desarrollo de las habilidades y a la comprensión musical son raramente citadas (McCarthy, 1993). Otros autores encuentran que la música es utilizada solo como entretenimiento; como medio de ilustrar otra materia, como descanso para otras materias o como música de ambiente (Bresler, 1993). Whitaker (1996) en un estudio cualitativo en el que la música se integraba sólo como recurso para el aprendizaje de otro material, encontró una progresiva devaluación del programa de música y del especialista de música.

Existen muchos factores que pueden afectar a la cantidad y la forma en que la música es incluida en la clase de infantil. En primer lugar, la actitud de los profesores hacia su propia habilidad musical y sus creencias y valoraciones hacia la inclusión de la música (Apfelstadt, 1989; Barry, 1992; Giles y Frego, 2004). El primero de ellos encontró que aquellos que habían vivido experiencias positivas hacia el canto en su infancia mostraban buena actitud en la edad adulta. Bresler (1993) obtuvo resultados similares, siendo muy importante la propia percepción de sus habilidades musicales. Aquellos que creían haber recibido poca formación musical y que había sido irrelevante en su formación universitaria la habían olvidado y por tanto se 
mostraban reticentes a utilizarlas en clase. Una gran mayoría se sentían poco cualificados para ofrecer a los niños las experiencias que necesitan. Además se encontró una gran discrepancia entre lo aprendido en los cursos y lo que los profesores consideran útil para sus clases (Giles y Frego, 2004; Saunders y Baker, 1991).

En general, los profesores no sienten la responsabilidad de incorporar objetivos musicales cuando incluyen actividades de tipo musical en su currículo (Byo, 1999; Giles y Frego, 2004). La integración cognitiva a través de la colaboración, suele ser más casual con momentos específicos muy concretos que como un hábito consistente entre el profesorado. La falta de tiempo suele ser la razón más frecuentemente dada para justificar la exclusión de las actividades musicales en sus clases (Bresler, 1993; Byo, 1999; Giles y Frego, 2004; McCarthy, 1993). Según estos autores esto también puede explicar el hecho de que los docentes sientan que están sacrificando menos tiempo académico si usan la música estrictamente para reforzar otras asignaturas que ellos consideran más importantes.

\section{EI especialista de Educación Musical}

Por parte del especialista de música, este trabajo en equipo interdisciplinar también suele resultar un punto controvertido ya que sienten que la música y su propia presencia puede ser reducida a un rol de servidumbre hacia otras áreas (Barry, 2008). Muchas de las experiencias realizadas en el aula muestran que, incluso en intentos bien intencionados de integrar las artes en los proyectos escolares, suelen acabar utilizando la música como una herramienta para enseñar otras áreas pero perdiendo el valor intrínseco musical (Mills, 2001). La falta de una persona experta en el ámbito musical lleva frecuentemente a realizar actividades sin desarrollo de las habilidades artísticas (Bresler, 1995), perdiendo en calidad de enseñanza. Los profesores especialistas a menudo muestran miedo a delegar excesiva responsabilidad hacia sus compañeros tutores ya que su conocimiento musical puede no ser suficiente para ofrecer un modelo apropiado a los niños, como en el canto afinado (Barry y Schons, 2005). Asimismo, si ya de por sí el tiempo dedicado a la música es escaso, existe la amenaza de que todavía se vea más limitado con este tipo de currículo (Cosenza, 2005). Esta situación provoca en los educadores musicales sentir emociones encontradas sobre el currículo interdisciplinar y el rol que la música puede desempeñar en el diseño y la ejecución de las tareas y actividades planteadas (Whitaker, 1996; Wiggins y Wiggins, 1997). Por un lado, defienden la importancia de la música dentro del currículo y su interés para la formación integral del ser humano, y por otra sienten la amenaza de que esta importancia sea minusvalorada y no plenamente aprovechada. 


\section{Integración de la música en la Educación Infantil}

Para una integración realmente satisfactoria de la música en el currículo a través del trabajo en equipo interdisciplinar de los docentes implicados, es necesario un proceso de adaptación continuado en el tiempo. Si los profesores tutores y especialistas en música han sido enseñados en métodos instruccionales tradicionales, no se les puede pedir que de repente cambien por completo su forma de enseñar (Barry y Schons, 2005; Corbett, Wilson, Noblit y McKinney, 2001). Los profesores necesitan tiempo para ganar su confianza en la planificación y enseñanza integradora sin perder la vista en los objetivos. Adecuar las oportunidades de desarrollo profesional y el tiempo de planificación colaborativa es importante para establecer nuevos canales de comunicación entre unos profesores y otros (Barry, 2008). McKean (2000) identificó como valores necesarios la colaboración, la identificación de los roles específicos de cada uno, la garantía de que los objetivos son consistentes y el mantenimiento de una relación recíproca entre los participantes. El entrenamiento continuado (Appel, 2006; Barry, 1998) y un tiempo regular de dedicación a la planificación ayuda a conseguir una colaboración significativa entre los especialistas y los tutores (Barry, 2008). Una vez que los profesores tutores han recibido el entrenamiento y han experimentado con la música, llegan a estar más interesados en ella como disciplina separada y valoran más el rol del especialista (Barry, 2008). Además muestran gran sorpresa al observar los cambios que presentan sus estudiantes al integrarla con sus áreas (Appel, 2006). Todo ello repercute también en la mayor valoración tanto de la música como del rol del especialista, al comprender de una manera más profunda su aportación al aprendizaje del alumno. Para los maestros de música puede servir para reducir el trabajo en solitario que muchas veces realizan en el ámbito escolar, participando de la organización compartida con los maestros generalistas (Miller, 1996). Además, los especialistas ocupan un puesto privilegiado en el trabajo colaborativo y en su ayuda a desarrollar las habilidades musicales de sus compañeros, con lo cual se aumentaría el aprendizaje musical aplicado en el aula de infantil. Pueden convertirse en coordinadores del equipo favoreciendo un trabajo realmente integrador y en el que la música ocupe el lugar que le corresponde (Snyder, 1996). Asimismo y entre otras labores pueden velar por el correcto entendimiento de todos los miembros de los objetivos pretendidos y ayudar a clarificar las posibles confusiones; conseguir el mayor consenso posible en la toma de decisiones; garantizar la expresión de todos los integrantes; buscar que los trabajos resulten gratificantes para todos los componentes y comprobar que el grupo dispone de recursos suficientes para afrontar los objetivos acordados, y si no, buscarlos (Bonals, 1996). 
La importancia del trabajo colaborativo interdisciplinar de docentes en la clase de infantil es esencial para conseguir una positiva y exitosa integración musical que mantenga y soporte la integridad de todas las áreas curriculares (Giles y Frego, 2004). Es por ello que el objetivo del estudio que en este documento se presenta es el de analizar la influencia del trabajo en equipo docente interdisciplinar que se constituyó entre docentes de educación infantil y especialista de música en este proyecto; examinando los parámetros que influyen en su funcionamiento y observando los beneficios de su aplicación en el proceso de enseñanza-aprendizaje del discurso lingüístico, verbal y musical, en el alumnado de infantil.

\section{Método}

\section{Participantes}

El equipo docente interdisciplinar objeto de este estudio ha estado constituido por tres maestras tutoras de $3^{\circ}$ curso de $2^{\circ}$ ciclo de Educación Infantil, a cargo de un total de 62 alumnos de entre 5 y 6 años, y la maestra especialista de música e investigadora. El equipo realizó cuatro reuniones conjuntas previas para definir unos objetivos didácticos comunes y consensuados entre todas las maestras, asentando las bases de funcionamiento del equipo docente. Posteriormente llevó a cabo, con periodicidad semanal, reuniones para programar conjuntamente las sesiones a desarrollar con el alumnado, así como evaluar su puesta en práctica y guiar la reprogramación en función de los resultados observados. La aplicación en el aula de todas las actividades con los estudiantes fue llevado a cabo por las tutoras, asistiendo la especialista de música a las sesiones como observadora no participante. Tras la puesta en práctica de todo el proyecto se realizó una última reunión del equipo docente con el fin de valorar todo el proceso vivido.

\section{Procedimiento e instrumentos}

Esta investigación está encuadrada dentro del enfoque cualitativo que permitió obtener información detallada de cómo las personas atribuyen significado a su actividad en contextos socioculturales definidos (Spindler y Hammond, 2000). El proceso de análisis se desarrolló dentro de la doble perspectiva de la investigación narrativa que facilita la reconstrucción y estructuración de la experiencia (Bruner, 2002) y de la investigación analítica cualitativa (Castanheira, Crawford, Dixon y Green, 2001) que implica el análisis de los diferentes materiales narrativos para llegar a su abstracción, conceptualización y elaboración de nuevas teorías (Gee y Green, 1998).

Para su realización se han analizado las reuniones realizadas semanalmente, grabadas en audio y transcritas para su estudio. Esta 
información se ha complementado con el análisis de los diarios de campo de cada uno de los integrantes del equipo colaborativo que han servido para el análisis y reflexión individual que después se ha puesto en común en las reuniones grupales. Además, se han realizado entrevistas semi-abiertas individuales a cada una de las maestras implicadas, tanto antes del proyecto como una vez finalizado. Todo este material ha sido guardado y analizado gracias al programa Atlas Ti, que ha favorecido la obtención de los resultados que a continuación se muestran.

\section{Discusión de resultados}

En el análisis de los resultados se ha pretendido definir los parámetros que con mayor énfasis han influido en el funcionamiento del trabajo en equipo colaborativo docente, así como observar los beneficios de su aplicación en el proyecto.

\section{Conocimientos y experiencias previas de cada docente}

El bagaje que cada docente poseía antes de iniciar este proyecto ha influido de manera significativa en el trabajo en equipo. Dentro de este apartado se pueden señalar tres ámbitos diferenciados:

- En el ámbito de la docencia general. Los años de experiencia docente han influido a la hora de proponer actividades ajustadas a las edades, capacidades y características concretas de los estudiantes. Cuanto mayor era la experiencia docente las actividades propuestas en una primera etapa del trabajo en equipo era más factibles y útiles en el proceso educativo vivido, tal y como muestra la investigadora en su diario de campo.

"Se nota la experiencia de M.L. a la hora de plantear las actividades y cómo diseñar las fichas. Los alumnos han sabido en todo momento seguir la clase y contestar las preguntas.”

- En el ámbito musical. Las vivencias de cada docente en relación con la música y los aprendizajes realizados a lo largo de su vida, tal y como señalaba Apfeslstadt (1989), han sido determinantes y han mediatizado las actitudes de dichas maestras sobre su propia habilidad, en la línea de los resultados encontrados por Barry (1992), Bresler (1993) y Giles y Frego (2004); marcando especialmente el punto de partida de esta investigación.

"Yo estoy dispuesta y me apetece hacer este proyecto, pero no tengo conocimientos musicales... Solo lo poquito que dimos en la carrera y eran cosas que no he podido aplicar en el aula... no sé si voy a poder ayudar mucho."

Como se observa con este ejemplo, en sus entrevistas indican que su formación preuniversitaria en el ámbito musical era inexistente y la universitaria era valorada de escasa y de poca utilidad, por falta de 
posibilidad de aplicación práctica en sus clases, como resaltaron en sus resultados autores como Saunders y Baker (1991) o Giles y Frego (2004).

- En el ámbito del trabajo en equipo. Dos de las maestras ya estaban habituadas al trabajo en equipo y habían trabajado juntas en cursos anteriores. Este hecho fue reconocido por todas las maestras como facilitador del funcionamiento del equipo en un inicio, al partir del bagaje de una dinámica previa.

\section{Interdependencia positiva entre los integrantes del grupo}

Las relaciones interpersonales positivas surgidas y ampliadas en el trabajo en equipo a lo largo del proyecto han reportado unos beneficios tanto personales como profesionales, en la línea de lo hallado por Bonals (1996) y López (2007), que han permitido mejorar la propia práctica educativa, tal y como indica Latorre (2003). En el equipo se ha observado un proceso en el que se ha partido de inhibiciones iniciales de ciertos miembros por miedo al error, que conllevaron desequilibrios iniciales en la participación y toma de decisiones en las primeras sesiones (Bonals, 1996) y que poco a poco se han conseguido equilibrar. Tres han sido los aspectos más valorados por las docentes para favorecer este equilibrio:

- La disposición física en círculo, con el mismo tipo de mesas y sillas para todos los integrantes, ya que en un lenguaje no verbal ha reforzado la idea de igualdad entre todos los miembros.

- El respeto y comprensión de todo el profesorado por las propuestas del resto de las compañeras, buscando reconducir y utilizar las ideas de las compañeras en los momentos en los que se encontraban más alejadas de la realidad del alumnado o de los objetivos del proyecto.

- La periodicidad de las reuniones, tal y como han hallado autores como Miller (1996). En esta investigación las reuniones semanales favorecieron la evaluación continua y cercana en el tiempo de lo sucedido en el aula, contrastando los resultados y datos obtenidos individualmente por cada maestra en su clase para la reformulación de los objetivos y actividades del proyecto.

\section{Responsabilidad compartida en el equipo}

Desde el inicio se buscó que las decisiones fueran consensuadas, siguiendo las directrices de Armengol (2002). Hay que indicar que al principio las maestras tutoras estaban a la espera de que la especialista de música sugiriera las canciones con las que trabajar y preparara las fichas. Tras las primeras reuniones y en especial tras la puesta en práctica con los alumnos de las sesiones iniciales, la responsabilidad de las tutoras aumentó significativamente, asumiendo por sí mismas la responsabilidad de las 
diferentes tareas. Así se deja ver en la propuesta de una de las maestras tras el trabajo real con el alumnado sobre la primera canción:

"Para la segunda canción me encargo yo de hacer la ficha y la traigo el próximo día para que la veáis.”

De manera similar se hace patente cuando la cuarta canción fue buscada y elegida por las maestras tutoras, sin depender de la especialista de música para ello. Además, las actividades fueron propuestas en su totalidad por ellas, siendo conscientes del grado de autonomía desarrollado:

“La evolución de las tutoras está siendo exponencial. Son ellas las que ya han propuesto para trabajar la canción Un barquito de cáscara de nuez y ya han comenzado con la lluvia de ideas sobre las actividades. Están muy bien secuenciadas con respecto a los aprendizajes que los alumnos han conseguido en las sesiones anteriores."

\section{Labor del especialista de música}

Los aspectos más destacados por las tutoras han sido la valoración de las ideas y actuaciones positivas de cada una de ellas, por la repercusión directa en su autoestima, especialmente en su competencia musical, y que ha conllevado cada vez a una mayor autonomía, como se observa en el siguiente comentario de una de las maestras:

"Las palabras de refuerzo de la especialista de música el otro día me hicieron darme cuenta de que era capaz de llevar a cabo el proyecto, pensaba al principio que no podría hacerlo.”

Otro de los puntos más valorados ha sido el de ofrecer los conocimientos y recursos musicales necesarios para afrontar los objetivos buscados.

"Este proyecto no hubiera sido posible sin la figura de la especialista de música. Ella nos ha dado la seguridad y las herramientas musicales necesarias para actuar con éxito.”

\section{Labor del centro}

De entre los diferentes aspectos que López (2007) resalta como dimensiones a ser reforzadas desde el Centro, los miembros del equipo han destacado dos. En primer lugar, la facilidad por parte del Equipo Directivo de que el grupo docente haya dispuesto de autonomía para programar aquellas actividades que ha considerado más interesantes para su grupo de alumnos. En segundo, la disponibilidad de tiempo para reunirse en el horario de permanencia en el Centro. Sin estas dos premisas el proyecto no hubiera podido llevarse a cabo.

Todos estos parámetros han influido en el funcionamiento del equipo docente interdisciplinar y han repercutido en ciertos beneficios en el proyecto, entre los que se destaca la producción de conocimiento, la 
formación y el desarrollo profesional (Lieberman, 1986; López, 2007). En referencia al primero, ha favorecido una producción de conocimiento lingüístico desde un enfoque enriquecido donde discurso verbal y musical se han visto mutuamente reforzados, tanto a nivel oral como escrito; valorando la importancia de la música en el proceso de enseñanza-aprendizaje del hecho lingüístico. Esta adquisición de conocimiento lingüístico, amplio y enriquecido por la complementariedad del discurso verbal y musical, se ha dado tanto en el alumnado como en el propio profesorado. Este hecho enlaza con el siguiente punto de formación, que se ha producido tanto en los maestros tutores por la adquisición de conocimientos musicales, desarrollando su sentimiento de competencia en el ámbito musical; como del especialista de música, tal y como sugiere Miller (1996), y de todos ellos por el enriquecimiento metodológico al compartir la forma de trabajo. Todo ello ha repercutido en una mejora en el desarrollo profesional docente, en la dirección apuntada por Stenhouse (1985). Se destaca el acceso a nuevas ideas, al aprendizaje y a la estimulación de la creatividad, en la línea de lo obtenido en su análisis López (2007). Asimismo, la reflexión individual y colectiva realizada en el proyecto les ha llevado a desarrollar su competencia como investigadores-actores en el proceso de mejora educativa (Desgagné, 1997). Todos estos beneficios han reportado una satisfacción no solo profesional sino también personal, que queda recogida con la declaración de intenciones de todas las maestras al afirmar:

"Volveré a repetir este proyecto."

\section{Conclusión}

En este estudio se ha analizado la influencia del trabajo en equipo docente interdisciplinar en la toma de conciencia del discurso lingüístico, verbal y musical, obtenida en el proceso de enseñanza-aprendizaje surgido entre las maestras tutoras y su alumnado de educación infantil. Se han examinado los parámetros que intervienen en su funcionamiento, observándose los beneficios de su aplicación. Como parámetros se han destacado tanto aquellos previos a la constitución del equipo, entre los que resaltan los conocimientos y experiencias previas de cada uno de los miembros; como los resultantes del proceso del trabajo colaborativo, como la interdependencia positiva y la responsabilidad compartida entre sus miembros. Asimismo se valora la influencia de la labor del especialista y del Centro. Una adecuada combinación de todos ellos ha favorecido unos resultados positivos en el proyecto. Tras el mismo, no solo los estudiantes sino también los tutores han sido más conscientes de todas las posibilidades que la música ofrecía para el desarrollo lingüístico integral y enriquecedor del alumnado; así como han desarrollado una mayor valoración hacia la música y hacia el especialista, en consonancia con los resultados de Barry 
(2008). La formación y el desarrollo profesional de todo el profesorado implicado, tanto maestras tutoras como especialista de música, también se ha visto favorecida, lo que muestra la repercusión positiva del trabajo en equipo colaborativo en sus integrantes. Se valora que el proyecto propuesto en equipo docente no solo es beneficioso para el alumnado sino también para el profesorado implicado en él.

\section{References:}

1. Antúnez, S. (1999). El trabajo en equipo de los profesores y profesoras: factor de calidad, necesidad y problema. El papel de los directivos escolares. Educar, 24, 89-110.

2. Apfelstadt, H. (1989). Do we have to sing? Factors affecting elementary education majors' attitudes toward singing. Update: Applications of Research in Music Education, 8(1), 24-27.

3. Appel, M. P. (2006). Arts integration across the curriculum. Leadership, 36(2), 14-17.

4. Armengol, C. (2002). El trabajo en equipo en los centros educativos. Barcelona: PRAXIS.

5. Barry, N. H. (1992). Music education in the elementary music methods class. Journal of Music Teacher Education, 2(1), 16-23.

6. Barry, N. H. (1998). Arts integration in the elementary school: Conference development and evaluation. Update: Applications of Research in Music Education, 17(1), 3-8.

7. Barry, N. H. (2008). The role of integrated curriculum in music teacher education. Journal of Music Teacher Education, 18(28), 2838.

8. Barry, N. H. y Schons, S. (2005). Integrated curriculum and the music teacher: A case study. Contributions to Music Education, 31(2), 57-72.

9. Bonals, J. (1996). El trabajo en equipo del profesorado. Barcelona: Graó.

10. Bresler, L. (1993). Music in a double bind: Instruction by nonspecialists in elementary schools. Council of Research in Music Education, 115, 1-13. Recuperado de http://faculty.education.illinois.edu/liora/sub_directory/pdf/MUSICD OUBLEBIND.pdf

11. Bresler, L. (1995). The subservient, co-equal, affective and social integration styles and their implications for the arts. Arts Education Policy Review, 96(5), 31-37. Recuperado de http://faculty.education.illinois.edu/liora/sub_directory/pdf/subservie nt.pdf 
12. Bruner, J. (2002). Making stories. Law, literature, life. Cambridge, MA \& London, UK: Harvard University Press.

13. Bryson, E. F. (1982). A study of the use of music activities by classroom teachers. Dissertation Abstracts International, 43, UMI No. 8228121.

14. Byo, S. J. (1999). Classroom teachers' and music specialists' perceived ability to implement the national standards for music education. Journal of Research in Music Education, 47(2), 111-123.

15. Castanheira, M. L., Crawford, T., Dixon, C. N. y Green, J. (2001). Interactional ethnography: An approach to studying the social construction of literacy practices. Linguistics and Education, 11(4), 353-400.

16. Corbett, D., Wilson, B., Noblit, G. y McKinney, M. (2001). The arts, identity and comprehensive education reform: A final report from the evaluation of the A+Schools Program. Winston-Salem, NC: Thomas $S$. Kenan Institute for the Arts.

17. Cosenza, G. (2005). Implications for music educators of an interdisciplinary curriculum. International Journal of Education \& the Arts, 6(9), 1-8. Recuperado de http://www.ijea.org/v6n9/v6n9.pdf

18. Desgagné, S. (1997). Le concept de recherche collaborative: l'idée d'un rapprochement entre chercheurs universitaires et praticiens enseignants. Revue des Sciences de l'Education, 23(2), 371-393.

19. Gee, J. P. y Green, J. L. (1998). Discourse analysis, learning, and social practice: a methodological study. Review of Research in Education, 23, 119-169. Recuperado de http://www.justinecassell.com/discourse/pdfs/gee-green98.pdf

20. Giles, A. M. y Frego, R. J. D. (2004). An inventory of music activities used by elementary classroom teachers: An exploratory study. Update: Applications of Research in Music Education, 22, 1322.

21. Latorre, A. (2003). La investigación-acción. Conocer y cambiar la práctica educativa. Barcelona: Graó.

22. Lieberman, A. (1986). Collaborative research: Working with, not working on. Educational Leadership, 43(45), 28-32.

23. López, A. (2007). Catorce ideas clave. El trabajo en equipo del profesorado. Barcelona: Graó.

24. McCarthy, W. G. (1985). Promoting language development through music. Academic Therapy, 21(2), 237-242.

25. McKean, B. (2000). Arts everyday: Classroom teacher's orientations toward arts education. Arts and Learning Research, 16(1), 177-194. 
26. Miller, B. A. (1996). Integrating Elementary General Music: A Collaborative Action Research Study. Bulletin of the Council for Research in Music Education, 130, 110-115.

27. Mills, S. W. (2001). The role of musical intelligence in a multiple intelligences focused elementary school. International Journal of Education \& the Arts, 2(4). Recuperado de http://www.ijea.org/v2n4/index.html

28. Saunders, T. C. y Baker, D. S. (1991). In-service classroom teachers' perceptions of useful music skills and understandings. Journal of Research in Music Education, 39(3), 248-261.

29. Snyder, S. (1996). Integrate with integrity: Music across the elementary curriculum. West Norwalk, CT: IDEAS Press.

30. Spindler, G. y Hammond, L. (2000). The use of anthropological methods in educational research: Two perspectives. Harvard Educational Review, 70(1), 39-48.

31. Stenhouse, L. (1985). El profesor como tema de investigación y desarrollo. Revista de Educación, 277, 43-54.

32. Whitaker, N. L. (1996). Elusive connections: Music integration and the elementary classroom. Bulletin of the Council for Research in Music Education, 130, 89-99.

33. Wiggins, J. y Wiggins, R. (1997). Integrating through conceptual connections. Music Educators' Journal, 83(4), 38-41. 